\title{
A Procedure to Construct Exact Solutions of Nonlinear Fractional Differential Equations
}

\author{
Özkan Güner ${ }^{1}$ and Adem C. Cevikel ${ }^{2}$ \\ ${ }^{1}$ Department of Management Information Systems, School of Applied Sciences, Dumlupinar University, 43100 Kutahya, Turkey \\ ${ }^{2}$ Department of Mathematics Education, Education Faculty, Yildiz Technical University, 34220 Istanbul, Turkey
}

Correspondence should be addressed to Adem C. Cevikel; acevikel@yildiz.edu.tr

Received 2 October 2013; Accepted 11 December 2013; Published 10 March 2014

Academic Editors: A. Kılıçman, S. S. Ray, and A. Secer

Copyright (C) 2014 Ö. Güner and A. C. Cevikel. This is an open access article distributed under the Creative Commons Attribution License, which permits unrestricted use, distribution, and reproduction in any medium, provided the original work is properly cited.

We use the fractional transformation to convert the nonlinear partial fractional differential equations with the nonlinear ordinary differential equations. The Exp-function method is extended to solve fractional partial differential equations in the sense of the modified Riemann-Liouville derivative. We apply the Exp-function method to the time fractional Sharma-Tasso-Olver equation, the space fractional Burgers equation, and the time fractional fmKdV equation. As a result, we obtain some new exact solutions.

\section{Introduction}

Fractional differential equations (FDEs) are generalizations of classical differential equations of integer order. Recently, fractional differential equations have gained much attention as they are widely used to describe various complex phenomena in various applications such as the fluid flow, signal processing, control theory, systems identification, finance and fractional dynamics, and physics. The fractional differential equations have been investigated by many researchers [1-3]. In recent decades, a large amount of literature has been provided to construct the exact solutions of fractional ordinary differential equations and fractional partial differential equations of physical interest. Many powerful and efficient methods have been proposed to obtain approximate solutions of fractional differential equations, such as the Adomian decomposition method $[4,5]$, the variational iteration method $[6,7]$, the homotopy analysis method $[8,9]$, the homotopy perturbation method $[10,11]$, and the differential transformation method [12-14]. The fractional subequation method [15-17], the first integral method [18], the Expfunction method $[19,20]$, and the $\left(\mathrm{G}^{\prime} / \mathrm{G}\right)$-expansion method [21-23] can be used to construct the exact solutions for some time and space fractional differential equations.

$\mathrm{He}$ and $\mathrm{Wu}$ [24] systematically proposed a new method in 2006, called the Exp-function method, to obtain exact solutions of nonlinear differential equations. The Expfunction method has been successfully applied to many kinds of nonlinear differential equations [25-28], such as highdimensional equations [29-31], variable-coefficient equations [32, 33], differential-difference equations [34, 35], and stochastic equations $[36,37]$.

The present paper investigates for the first time the applicability and effectiveness of the Exp-function method on fractional nonlinear partial differential equations.

\section{The Modified Riemann-Liouville Derivative}

Jumarie proposed a modified Riemann-Liouville derivative. With this kind of fractional derivative and some useful formulas, we can convert fractional differential equations into integer-order differential equations by variable transformation in [38].

In this section, we firstly give some properties and definitions of the modified Riemann-Liouville derivative which are used further in this paper.

Assume that $f: R \rightarrow R, x \rightarrow f(x)$ denote a continuous (but not necessarily differentiable) function. The Jumarie modified Riemann-Liouville derivative of order $\alpha$ is defined by the expression 


$$
D_{x}^{\alpha} f(x)= \begin{cases}\frac{1}{\Gamma(-\alpha)} \int_{0}^{x}(x-\xi)^{-\alpha-1}[f(\xi)-f(0)] d \xi, & \alpha<0 \\ \frac{1}{\Gamma(1-\alpha)} \frac{d}{d x} \int_{0}^{x}(x-\xi)^{-\alpha}[f(\xi)-f(0)] d \xi, & 0<\alpha<1 \\ \left(f^{(n)}(x)\right)^{(\alpha-n)}, & n \leq \alpha \leq n+1, n \geq 1 .\end{cases}
$$

A few properties of the fractional modified RiemannLiouville derivative were summarized and three famous formulas of them are

$$
\begin{gathered}
D_{x}^{\alpha} x^{\gamma}=\frac{\Gamma(1+\gamma)}{\Gamma(1+\gamma-\alpha)} x^{\gamma-\alpha}, \quad \gamma>0, \\
D_{x}^{\alpha}(u(x) v(x))=v(x) D_{x}^{\alpha} u(x)+u(x) D_{x}^{\alpha} v(x), \\
D_{x}^{\alpha} f[u(x)]=f_{u}^{\prime}(u) D_{x}^{\alpha} u(x)=D_{u}^{\alpha} f(u)\left(u_{x}^{\prime}\right)^{\alpha},
\end{gathered}
$$

which are direct consequences of the equality

$$
d^{\alpha} x(t)=\Gamma(1+\alpha) d x(t) .
$$

Secondly, let us consider the time fractional differential equation with independent variables $x=\left(x_{1}, x_{2}, \ldots, x_{m}, t\right)$ and a dependent variable $u$ :

$$
F\left(u, D_{t}^{\alpha} u, u_{x_{1}}, u_{x_{2}}, u_{x_{3}}, D_{t}^{2 \alpha} u, u_{x_{1} x_{1}}, u_{x_{2} x_{2}}, u_{x_{3} x_{3}}, \ldots\right)=0
$$

Using the fractional variable transformation

$$
\begin{gathered}
U(\xi)=u\left(x_{1}, x_{2}, \ldots, x_{m}, t\right) \\
\xi=x_{1}+l_{1} x_{2}+\cdots+l_{m-1} x_{m}+\frac{\lambda t^{\alpha}}{\Gamma(1+\alpha)},
\end{gathered}
$$

where $l_{i}$ and $\lambda$ are constants to be determined later. Similarly, let us consider the space fractional differential equation with independent variables $x=\left(x_{1}, x_{2}, \ldots, x_{m}, t\right)$ and a dependent variable $u$ :

$$
F\left(u, u_{t}, D_{x_{1}}^{\beta} u, u_{x_{2}}, u_{x_{3}}, D_{x_{1}}^{2 \beta} u, u_{x_{1} x_{1}}, u_{x_{2} x_{2}}, u_{x_{3} x_{3}}, \ldots\right)=0 .
$$

Next, using the fractional variable transformation

$$
\begin{gathered}
U(\xi)=u\left(x_{1}, x_{2}, \ldots, x_{m}, t\right), \\
\xi=\frac{\lambda x_{1}^{\beta}}{\Gamma(1+\beta)}+l_{1} x_{2}+\cdots+l_{m-1} x_{m}+l_{m} t
\end{gathered}
$$

where $l_{i}$ and $\lambda$ are constants to be determined later.

The fractional differential equation (6) is reduced to a nonlinear ordinary differential equation

$$
H=\left(U(\xi), U^{\prime}(\xi), U^{\prime \prime}(\xi), \ldots\right)
$$

where “»" $=d / d \xi$.

\section{Description of the Exp-Function Method}

We consider the general nonlinear ordinary differential equation in (8). According to Exp-function method, we assume that the wave solution can be expressed in the following form [24]:

$$
U(\xi)=\frac{\sum_{n=-c}^{d} a_{n} \exp [n \xi]}{\sum_{m=-p}^{q} b_{m} \exp [m \xi]},
$$

where $p, q, c$, and $d$ are positive integers which are known to be further determined and $a_{n}$ and $b_{m}$ are unknown constants. We can rewrite (9) in the following equivalent form:

$$
U(\xi)=\frac{a_{-c} \exp [-c \xi]+\cdots+a_{d} \exp [d \xi]}{b_{-p} \exp [-p \xi]+\cdots+b_{q} \exp [q \xi]}
$$

This equivalent formulation plays an important and fundamental part for finding the analytic solution of problems. To determine the value of $c$ and $p$, we balance the linear term of the highest order of (8) with the highest degree nonlinear term. Similarly, to determine the value of $d$ and $q$, we balance the linear term of the lowest order of (8) with the lowest degree nonlinear term.

We suppose that the solution in (8) can be expressed as

$$
U(\xi)=\sum_{i=1}^{n} a_{i} \phi^{i}
$$

where $\phi$ is the solution of the auxiliary equation $\phi^{\prime}=\alpha+\beta \phi+$ $\gamma \phi^{2}$. In a similar way, $\phi$ can be expressed in (11).

Theorem 1. Suppose that $U^{(r)}$ and $U^{s}$ are, respectively, the highest order linear term and the highest degree nonlinear term of a nonlinear ODE, where $r$ and $s$ are both positive integers. Then the balancing procedure using the Exp-function ansatz $U(\xi)=\sum_{n=-c}^{d} a_{n} \exp (n \xi) / \sum_{m=-p}^{q} b_{m} \exp (m \xi)$ leads to $d=q$ and $c=p$ and $\forall r \geq 1, \forall s \geq 2$ [39].

To show the efficiency of the method described in the previous part, we present some FDEs examples.

\section{The Time Fractional Sharma-Tasso-Olver Equation}

We consider the nonlinear fractional Sharma-Tasso-Olver equation [40]

$$
\begin{array}{r}
D_{t}^{\alpha} u+3 a u_{x}^{2}+3 a u^{2} u_{x}+3 a u u_{x x}+a u_{x x x}=0, \\
t>0, \quad 0<\alpha \leq 1,
\end{array}
$$


subject to the initial condition

$$
u(x, 0)=-\sqrt{2 B_{0}} \tan \left(\frac{\sqrt{2 B_{0}}}{2} x\right),
$$

where $a$ and $B_{0}$ are arbitrary constants and $\alpha$ is a parameter describing the order of the fractional time derivative. The function $u(x, t)$ is assumed to be a causal function of time.

For our purpose, we introduce the following transformations:

$$
u(x, t)=U(\xi), \quad \xi=x-\frac{\lambda t^{\alpha}}{\Gamma(1+\alpha)},
$$

where $\lambda$ is a constant.

Substituting (14) into (12), we can know that (12) reduced into an ODE

$$
-\lambda U^{\prime}+3 a\left(U^{\prime}\right)^{2}+3 a U^{2} U^{\prime}+3 a U U^{\prime \prime}+a U^{\prime \prime \prime}=0,
$$

where " $U^{\prime \prime}=d U / d \xi$.

Integrating (15) with respect to $\xi$ yields

$$
\xi_{0}-\lambda U+3 a U U^{\prime}+a U^{3}+a U^{\prime \prime}=0,
$$

where $\xi_{0}$ is a constant of integration.

Here take notice of the nonlinear term in (16), and we can balance $U^{\prime \prime}$ and $U^{3}$ by the idea of the Exp-function method [24] to determine the values of $p, q, c$, and $d$. By simple calculation, we have

$$
\begin{aligned}
U^{3} & =\frac{c_{1} \exp [-(3 c+p) \xi]+\cdots}{c_{2} \exp [-4 p \xi]+\cdots}, \\
U^{\prime \prime} & =\frac{c_{3} \exp [-(3 p+c) \xi]+\cdots}{c_{4} \exp [-4 p \xi]+\cdots},
\end{aligned}
$$

where $c_{i}$ are determined coefficients only for simplicity. Balancing the highest order of Exp-function in (17) we have

$$
-(3 p+c)=-(3 c+p),
$$

which leads to the result

$$
p=c
$$

Similarly to determine values of $d$ and $q$, we balance the linear term of the lowest order in (16):

$$
\begin{aligned}
& U^{\prime \prime}=\frac{\cdots+d_{1} \exp [(3 q+d) \xi]}{\cdots+d_{2} \exp [4 q \xi]}, \\
& U^{3}=\frac{\cdots+d_{3} \exp [(3 d+q) \xi]}{\cdots+d_{4} \exp [4 q \xi]},
\end{aligned}
$$

where $d_{i}$ are determined coefficients only for simplicity. From (20), we obtain

$$
3 q+d=3 d+q
$$

and this gives

$$
q=d
$$

For simplicity, we set $p=c=1$ and $q=d=1$, so (10) reduces to

$$
U(\xi)=\frac{a_{1} \exp (\xi)+a_{0}+a_{-1} \exp (-\xi)}{b_{1} \exp (\xi)+b_{0}+b_{-1} \exp (-\xi)} .
$$

Substituting (23) into (16) and by the help of symbolic computation, we have

$$
\begin{aligned}
\frac{1}{A} & {\left[R_{3} \exp (3 \xi)+R_{2} \exp (2 \xi)+R_{1} \exp (\xi)+R_{0}\right.} \\
& \left.+R_{-1} \exp (-\xi)+R_{-2} \exp (-2 \xi)+R_{-3} \exp (-3 \xi)\right] \\
& =0
\end{aligned}
$$

where

$$
\begin{aligned}
& A=\left(b_{-1} \exp (-\xi)+b_{0}+b_{1} \exp (\xi)\right)^{3}, \\
& R_{3}=-\lambda a_{1} b_{1}^{2}+a a_{1}^{3}+\xi_{0} b_{1}^{3} \text {, } \\
& R_{2}=a a_{0} b_{1}^{2}-\lambda a_{0} b_{1}^{2}+3 a a_{1}^{2} b_{0}+3 a a_{1}^{2} a_{0} \\
& +3 \xi_{0} b_{1}^{2} b_{0}-a a_{1} b_{1} b_{0}-2 \lambda a_{1} b_{1} b_{0}-3 a a_{1} a_{0} b_{1}, \\
& R_{1}=-2 \lambda a_{0} b_{1} b_{0}+3 a a_{0} a_{1} b_{0}-a a_{0} b_{1} b_{0}-3 a a_{0}^{2} b_{1} \\
& +3 a a_{1} a_{0}^{2}+a a_{1} b_{0}^{2}+3 \xi_{0} b_{1} b_{0}^{2}-\lambda a_{1} b_{0}^{2} \\
& +3 \xi_{0} b_{1}^{2} b_{-1}-\lambda a_{-1} b_{1}^{2}+6 a a_{1}^{2} b_{-1} \\
& +3 a a_{1}^{2} a_{-1}+4 a a_{-1} b_{1}^{2}-2 \lambda a_{1} b_{1} b_{-1} \\
& -6 a a_{1} a_{-1} b_{1}-4 a a_{1} b_{1} b_{-1} \text {, } \\
& R_{0}=3 a a_{-1} b_{1} b_{0}+\xi_{0} b_{0}^{3}+a a_{0}^{3}-\lambda a_{0} b_{0}^{2} \\
& -2 \lambda a_{-1} b_{1} b_{0}+9 a a_{1} a_{0} b_{-1}-9 a a_{0} a_{-1} b_{1} \\
& +6 a a_{1} a_{0} a_{-1}+3 a a_{1} b_{0} b_{-1} \text {, } \\
& R_{-1}=-2 \lambda a_{0} b_{-1} b_{0}-3 a a_{0} a_{-1} b_{0}-a a_{0} b_{-1} b_{0} \\
& -\lambda a_{-1} b_{0}^{2}+3 a a_{0}^{2} b_{-1}+3 a a_{0}^{2} a_{-1} \\
& +a a_{-1} b_{0}^{2}+3 \xi_{0} b_{0}^{2} b_{-1}+3 \xi_{0} b_{1} b_{-1}^{2} \\
& -\lambda a_{1} b_{-1}^{2}-6 a a_{-1}^{2} b_{1}+3 a a_{1} a_{-1}^{2} \\
& +4 a a_{1} b_{-1}^{2}-2 \lambda a_{-1} b_{1} b_{-1}+6 a a_{-1} a_{1} b_{-1} \\
& -4 a a_{-1} b_{1} b_{-1} \text {, } \\
& R_{-2}=a a_{0} b_{-1}^{2}-\lambda a_{0} b_{-1}^{2}-3 a a_{-1}^{2} b_{0} \\
& +3 a a_{0} a_{-1}^{2}+3 \xi_{0} b_{0} b_{-1}^{2}-2 \lambda a_{-1} b_{0} b_{-1} \\
& +3 a a_{-1} a_{0} b_{-1}-a a_{-1} b_{0} b_{-1} \\
& R_{-3}=-\lambda a_{-1} b_{-1}^{2}+\xi_{0} b_{-1}^{3}+a a_{-1}^{3} \text {. }
\end{aligned}
$$


Solving this system of algebraic equations by using symbolic computation, we obtain the following results.

Case 1. We have

$$
\begin{gathered}
a_{0}=0, \quad b_{-1}=-\frac{a_{-1}}{2}, \quad b_{0}=0, \\
b_{1}=\frac{a_{1}}{2}, \quad \xi_{0}=0, \\
\lambda=\lambda, \quad a=\frac{\lambda}{4},
\end{gathered}
$$

where $a_{-1}$ and $a_{1}$ are free parameters. Substituting these results into (23), we obtain the following exact solution:

$$
\begin{aligned}
u(x, t)= & \left(a_{1} \exp \left(x-\frac{\lambda t^{\alpha}}{\Gamma(1+\alpha)}\right)\right. \\
& \left.+a_{-1} \exp \left(-\left(x-\frac{\lambda t^{\alpha}}{\Gamma(1+\alpha)}\right)\right)\right) \\
& \times\left(\frac{a_{1}}{2} \exp \left(x-\frac{\lambda t^{\alpha}}{\Gamma(1+\alpha)}\right)\right. \\
& \left.\quad-\frac{a_{-1}}{2} \exp \left(-\left(x-\frac{\lambda t^{\alpha}}{\Gamma(1+\alpha)}\right)\right)\right)^{-1} .
\end{aligned}
$$

If we set $a_{1}=2$ and $a_{-1}=-2,(27)$ becomes

$$
u(x, t)=\tanh \left(x-\frac{\lambda t^{\alpha}}{\Gamma(1+\alpha)}\right)
$$

which is the other exact solution of the fractional SharmaTasso-Olver equation.

If we set $a_{1}=a_{-1}=2$, (27) becomes

$$
u(x, t)=\operatorname{coth}\left(x-\frac{\lambda t^{\alpha}}{\Gamma(1+\alpha)}\right)
$$

which is the other exact solution of the fractional SharmaTasso-Olver equation.

Case 2. We have

$$
\begin{gathered}
a_{0}=0, \quad b_{-1}=b_{-1}, \quad b_{0}=0, \\
b_{1}=\frac{a_{1} b_{-1}}{\left(a_{-1}+2 b_{-1}\right)}, \quad \xi_{0}=\xi_{0}, \\
\lambda=\frac{\xi_{0} b_{-1}\left(3 a_{-1}^{2}+6 a_{-1} b_{-1}+4 b_{-1}^{2}\right)}{2 a_{-1}\left(a_{-1}+2 b_{-1}\right)\left(a_{-1}+b_{-1}\right)}, \\
a=\frac{b_{-1}^{3} \xi_{0}}{2 a_{-1}\left(a_{-1}+2 b_{-1}\right)\left(a_{-1}+b_{-1}\right)},
\end{gathered}
$$

where $a_{-1}$ and $b_{-1}$ are free parameters. Substituting these results into (23), we obtain the following exact solution:

$$
\begin{aligned}
u(x, t)= & \left(a_{1} \exp \left(x-\frac{\lambda t^{\alpha}}{\Gamma(1+\alpha)}\right)\right. \\
& \left.+a_{-1} \exp \left(-\left(x-\frac{\lambda t^{\alpha}}{\Gamma(1+\alpha)}\right)\right)\right) \\
& \times\left(\frac{a_{1} b_{-1}}{\left(a_{-1}+2 b_{-1}\right)} \exp \left(x-\frac{\lambda t^{\alpha}}{\Gamma(1+\alpha)}\right)\right. \\
& \left.\quad+b_{-1} \exp \left(-\left(x-\frac{\lambda t^{\alpha}}{\Gamma(1+\alpha)}\right)\right)\right)^{-1} .
\end{aligned}
$$

Comparing our results with the results $[18,19]$, it can be seen that our results are new to our best knowledge.

\section{The Space Fractional Burgers Equation}

We consider the space fractional Burgers equation [41]

$$
\begin{array}{r}
\frac{\partial u}{\partial t}+u \frac{\partial u}{\partial x}-k \frac{\partial^{2} u}{\partial x^{2}}+n \frac{\partial^{\beta} u}{\partial x^{\beta}}=0, \\
x, t>0, \quad 0<\beta \leq 1,
\end{array}
$$

with the following initial value problem:

$$
u(0, t)=0, \quad u_{x}(0, t)=\frac{1}{t}-\frac{\pi^{2}}{2 k t^{2}},
$$

where $k$ and $n$ are arbitrary constants and $\beta$ is a parameter describing the order of the fractional space derivative. The function $u(x, t)$ is assumed to be a causal function of time.

For our purpose, we introduce the following transformations:

$$
u(x, t)=U(\xi), \quad \xi=\frac{\lambda x^{\beta}}{\Gamma(1+\beta)}-c t
$$

where $\lambda$ is a constant.

Substituting (34) into (32), we can know that (32) reduced into an ODE

$$
-c U^{\prime}+\lambda U U^{\prime}-k \lambda^{2} U^{\prime \prime}+n \lambda U^{\prime}=0,
$$

where " $U$ " $=d U / d \xi$.

Integrating (35) with respect to $\xi$ yields

$$
(\lambda n-c) U+\lambda \frac{U^{2}}{2}-k \lambda^{2} U^{\prime}+\xi_{0}=0,
$$

where $\xi_{0}$ is a constant of integration.

Here take notice of the nonlinear term in (36), and we can balance $U^{\prime}$ and $U^{2}$ by the idea of the Exp-function method [24] to determine the values of $p, q, c$, and $d$. By simple calculation, we have

$$
\begin{gathered}
U^{\prime}=\frac{c_{1} \exp [-(c+p) \xi]+\cdots}{c_{2} \exp [-2 p \xi]+\cdots}, \\
U^{2}=\frac{c_{3} \exp [-2 c \xi]+\cdots}{c_{4} \exp [-2 p \xi]+\cdots},
\end{gathered}
$$


where $c_{i}$ are determined coefficients only for simplicity. Balancing the highest order of Exp-function in (37) we have

$$
-(p+c)=-2 c
$$

which leads to the result

$$
p=c
$$

Similarly to determine values of $d$ and $q$, we balance the linear term of the lowest order in (36):

$$
\begin{gathered}
U^{\prime}=\frac{\cdots+d_{1} \exp [(q+d) \xi]}{\cdots+d_{2} \exp [2 q \xi]}, \\
U^{2}=\frac{\cdots+d_{3} \exp [2 d \xi]}{\cdots+d_{4} \exp [2 q \xi]},
\end{gathered}
$$

where $d_{i}$ are determined coefficients only for simplicity. From (40), we obtain

$$
q+d=2 d
$$

and this gives

$$
q=d
$$

For simplicity, we set $p=c=1$ and $q=d=1$, so (10) reduces to

$$
U(\xi)=\frac{a_{1} \exp (\xi)+a_{0}+a_{-1} \exp (-\xi)}{b_{1} \exp (\xi)+b_{0}+b_{-1} \exp (-\xi)}
$$

Substituting (43) into (36) and by the help of computation, we have

$$
\begin{aligned}
& \frac{1}{A}\left[R_{2} \exp (2 \xi)+R_{1} \exp (\xi)+R_{0}\right. \\
& \left.\quad+R_{-1} \exp (-\xi)+R_{-2} \exp (-2 \xi)\right]=0
\end{aligned}
$$

where

$$
\begin{aligned}
A= & \left(b_{-1} \exp (-\xi)+b_{0}+b_{1} \exp (\xi)\right)^{2}, \\
R_{2}= & \frac{\lambda}{2} a_{1}^{2}+\xi_{0} b_{1}^{2}-c a_{1} b_{1}+n \lambda a_{1} b_{1}, \\
R_{1}= & -c a_{1} b_{0}+2 \xi_{0} b_{1} b_{0}+\lambda a_{1} a_{0}-c a_{0} b_{1} \\
& +n \lambda a_{0} b_{1}+k \lambda^{2} a_{0} b_{1} \\
& +n \lambda a_{1} b_{0}-k \lambda^{2} a_{1} b_{0}, \\
R_{0}= & -2 k \lambda^{2} a_{1} b_{-1}+2 k \lambda^{2} a_{-1} b_{1}+n \lambda a_{1} b_{-1} \\
& +n \lambda a_{0} b_{0}+n \lambda b_{1} a_{-1}+\frac{1}{2} \lambda a_{0}^{2} \\
& -c a_{0} b_{0}+\xi_{0} b_{0}^{2}+2 \xi_{0} b_{1} b_{-1} \\
& -c a_{1} b_{-1}-c b_{1} a_{-1}+\lambda b a_{1} a_{-1},
\end{aligned}
$$

$$
\begin{aligned}
R_{-1}= & -c a_{-1} b_{0}-c a_{0} b_{-1}+2 \xi_{0} b_{0} b_{-1} \\
& +\lambda a_{0} a_{-1}-k \lambda^{2} a_{0} b_{-1}+n \lambda a_{-1} b_{0} \\
& +n \lambda a_{0} b_{-1}+k \lambda^{2} a_{-1} b_{0}
\end{aligned}
$$

$$
R_{-2}=\frac{1}{2} \lambda a_{-1}^{2}+\xi_{0} b_{-1}^{2}-c a_{-1} b_{-1}+n \lambda a_{-1} b_{-1} \text {. }
$$

Solving this system of algebraic equations by using symbolic computation, we obtain the following results.

Case 1. We have

$$
\begin{gathered}
a_{1}=\frac{b_{1}\left(-4 k \lambda b_{-1}+a_{-1}\right)}{b_{-1}}, \quad a_{0}=0, \\
k=k, \quad b_{1}=b_{1}, \quad b_{0}=0, \quad \lambda=\lambda, \\
c=\frac{\lambda\left(-2 k \lambda b_{-1}+n b_{-1}+a_{-1}\right)}{b_{-1}},
\end{gathered}
$$

$$
\xi_{0}=\frac{\lambda a_{-1}\left(-4 k \lambda b_{-1}+a_{-1}\right)}{2 b_{-1}^{2}}, \quad n=n
$$

where $a_{-1}, b_{1}$, and $b_{-1}$ are free parameters. Substituting these results into (43), we get the following exact solution:

$$
\begin{aligned}
u(x, t)= & \left(\frac{b_{1}\left(-4 k \lambda b_{-1}+a_{-1}\right)}{b_{-1}} \exp \left(\frac{\lambda x^{\beta}}{\Gamma(1+\beta)}-c t\right)\right. \\
& \left.+a_{-1} \exp \left(-\left(\frac{\lambda x^{\beta}}{\Gamma(1+\beta)}-c t\right)\right)\right) \\
& \times\left(b_{1} \exp \left(\frac{\lambda x^{\beta}}{\Gamma(1+\beta)}-c t\right)\right. \\
& \left.+b_{-1} \exp \left(-\left(\frac{\lambda x^{\beta}}{\Gamma(1+\beta)}-c t\right)\right)\right)^{-1},
\end{aligned}
$$

which is the exact solution of the space fractional Burgers equation.

Case 2. We have

$$
\begin{gathered}
a_{-1}=0, \quad a_{0}=-\frac{b_{0}\left(\lambda a_{1}+2 n \lambda b_{1}-2 c b_{1}\right)}{\lambda b_{1}}, \\
k=\frac{c b_{1}-\lambda a_{1}-n \lambda b_{1}}{\lambda^{2} b_{1}}, \quad b_{-1}=0, \\
b_{0}=b_{0}, \quad \lambda=\lambda, \quad c=c, \\
\xi_{0}=-\frac{a_{1}\left(\lambda a_{1}+2 n \lambda b_{1}-2 c b_{1}\right)}{2 b_{1}^{2}}, \quad n=n,
\end{gathered}
$$


where $a_{1}, b_{0}$, and $b_{1}$ are free parameters. Substituting these results into (43), we obtain the following exact solution:

$$
\begin{aligned}
u(x, t)= & \left(a_{1} \exp \left(\frac{\lambda x^{\beta}}{\Gamma(1+\beta)}-c t\right)\right. \\
& \left.-\frac{b_{0}\left(\lambda a_{1}+2 n \lambda b_{1}-2 c b_{1}\right)}{\lambda b_{1}}\right) \\
& \times\left(b_{1} \exp \left(\frac{\lambda x^{\beta}}{\Gamma(1+\beta)}-c t\right)+b_{0}\right)^{-1},
\end{aligned}
$$

which is the exact solution of the space fractional Burgers equation.

Case 3. We have

$$
\begin{aligned}
& a_{1}=-\left(a_{-1}^{2} b_{0}^{2}-2 a_{-1} b_{0}^{2} k \lambda b_{-1}-2 a_{0} b_{0} a_{-1} b_{-1}\right. \\
& \left.+2 a_{0} b_{0} b_{-1}^{2} k \lambda+a_{0}^{2} b_{-1}^{2}\right)\left(a_{-1}-2 k \lambda b_{-1}\right) \\
& \times\left(4 b_{-1}^{4} k^{2} \lambda^{2}\right)^{-1}, \\
& b_{1}=-\left(a_{-1}^{2} b_{0}^{2}-2 a_{-1} b_{0}^{2} k \lambda b_{-1}-2 a_{0} b_{0} a_{-1} b_{-1}\right. \\
& \left.+2 a_{0} b_{0} b_{-1}^{2} k \lambda+a_{0}^{2} b_{-1}^{2}\right)\left(4 b_{-1}^{3} k^{2} \lambda^{2}\right)^{-1}, \\
& a_{0}=a_{0}, \quad a_{-1}=a_{-1}, \quad b_{0}=b_{0}, \quad b_{-1}=b_{-1} \text {, } \\
& c=\frac{\lambda\left(a_{-1}-k \lambda b_{-1}+n b_{-1}\right)}{b_{-1}}, \quad k=k, \quad \lambda=\lambda, \\
& n=n, \quad \xi_{0}=\frac{\lambda a_{-1}\left(a_{-1}-2 k \lambda b_{-1}\right)}{2 b_{-1}^{2}},
\end{aligned}
$$

where $a_{0}, a_{-1}, b_{0}$, and $b_{-1}$ are free parameters. Substituting these results into (43), we get the following exact solution:

$$
\begin{aligned}
u(x, t)=( & -\left(a_{-1}^{2} b_{0}^{2}-2 a_{-1} b_{0}^{2} k \lambda b_{-1}-2 a_{0} b_{0} a_{-1} b_{-1}\right. \\
& \left.\quad+2 a_{0} b_{0} b_{-1}^{2} k \lambda+a_{0}^{2} b_{-1}^{2}\right) \\
& \times\left(a_{-1}-2 k \lambda b_{-1}\right)\left(4 b_{-1}^{4} k^{2} \lambda^{2}\right)^{-1} \\
& \times \exp \left(\frac{\lambda x^{\beta}}{\Gamma(1+\beta)}-c t\right)+a_{0} \\
& \left.+a_{-1} \exp \left(-\left(\frac{\lambda x^{\beta}}{\Gamma(1+\beta)}-c t\right)\right)\right)
\end{aligned}
$$

$$
\begin{gathered}
\times\left(-\left(a_{-1}^{2} b_{0}^{2}-2 a_{-1} b_{0}^{2} k \lambda b_{-1}-2 a_{0} b_{0} a_{-1} b_{-1}\right.\right. \\
\left.\quad+2 a_{0} b_{0} b_{-1}^{2} k \lambda+a_{0}^{2} b_{-1}^{2}\right) \\
\times\left(4 b_{-1}^{3} k^{2} \lambda^{2}\right)^{-1} \\
\times \exp \left(\frac{\lambda x^{\beta}}{\Gamma(1+\beta)}-c t\right)+b_{0}+b_{-1} \\
\left.\quad \times \exp \left(-\left(\frac{\lambda x^{\beta}}{\Gamma(1+\beta)}-c t\right)\right)\right)^{-1}
\end{gathered}
$$

which is the exact solution of the space fractional Burgers equation.

The obtained solutions for the space fractional Burgers equation are new to our best knowledge.

\section{The Time Fractional fmKdV Equation}

We consider the following fractional time fractional fmKdV equation [42]:

$$
\begin{array}{r}
D_{t}^{\alpha} u+u^{2} u_{x}+u_{x x x}=0, \\
t>0, \quad 0<\alpha \leq 1,
\end{array}
$$

with the initial conditions as

$$
u(x, 0)=\frac{4 \sqrt{2} k \sin ^{2}(k x)}{3-\sin ^{2}(k x)},
$$

where $k$ is an arbitrary constant and $\alpha$ is a parameter describing the order of the fractional time derivative.

For our purpose, we introduce the following transformations

$$
u(x, t)=U(\xi), \quad \xi=c x-\frac{\lambda t^{\alpha}}{\Gamma(1+\alpha)},
$$

where $\lambda$ and $c$ are constants.

Substituting (54) into (52), we can know that (52) reduced into an ODE

$$
-\lambda U^{\prime}+c U^{2} U^{\prime}+c^{3} U^{\prime \prime \prime}=0
$$

where " $U$ " $=d U / d \xi$.

By using the ansatz (55), for the linear term of highest order $U^{\prime \prime \prime}$ with the highest order and the nonlinear term $U^{2} U^{\prime}$, balancing $U^{\prime \prime \prime}$ with $U^{2} U^{\prime}$ in (55) gives

$$
\begin{aligned}
U^{\prime \prime \prime} & =\frac{c_{1} \exp [-(7 p+c) \xi]+\cdots}{c_{2} \exp [-8 p \xi]+\cdots} \\
& =\frac{c_{1} \exp [-(3 p+c) \xi]+\cdots}{c_{2} \exp [-4 p \xi]+\cdots}, \\
U^{2} U^{\prime} & =\frac{c_{3} \exp [-(p+3 c) \xi]+\cdots}{c_{4} \exp [-4 p \xi]+\cdots},
\end{aligned}
$$


where $c_{i}$ are determined coefficients only for simplicity. Balancing the highest order of Exp-function in (56) we have

$$
-(3 p+c)=-(3 c+p),
$$

which leads to the result

$$
p=c .
$$

Similarly to determine values of $d$ and $q$, we balance the linear term of the lowest order in (55):

$$
\begin{gathered}
U^{\prime \prime \prime}=\frac{\cdots+d_{1} \exp [(3 q+d) \xi]}{\cdots+d_{2} \exp [4 q \xi]}, \\
U^{2} U^{\prime}=\frac{\cdots+d_{3} \exp [(3 d+q) \xi]}{\cdots+d_{4} \exp [4 q \xi]},
\end{gathered}
$$

where $d_{i}$ are determined coefficients only for simplicity. From (59), we obtain

$$
3 q+d=3 d+q
$$

and this gives

$$
q=d
$$

For simplicity, we set $p=c=1$ and $q=d=1$, so (10) reduces to

$$
U(\xi)=\frac{a_{1} \exp (\xi)+a_{0}+a_{-1} \exp (-\xi)}{b_{1} \exp (\xi)+b_{0}+b_{-1} \exp (-\xi)}
$$

Substituting (62) into (55) and by the help of computation, we have

$$
\begin{aligned}
\frac{1}{A}[ & R_{3} \exp (3 \xi)+R_{2} \exp (2 \xi)+R_{1} \exp (\xi)+R_{0} \\
& +R_{-1} \exp (-\xi)+R_{-2} \exp (-2 \xi) \\
& \left.+R_{-3} \exp (-3 \xi)\right]=0,
\end{aligned}
$$

where

$$
\begin{aligned}
A= & \left(b_{-1} \exp (-\xi)+b_{0}+b_{1} \exp (\xi)\right)^{4}, \\
R_{3}= & \lambda a_{0} b_{1}^{3}-c^{3} a_{0} b_{1}^{3}+c a_{1}^{3} b_{0} \\
& -\lambda a_{1} b_{1}^{2} b_{0}-c a_{1}^{2} a_{0} b_{1}+c^{3} a_{1} b_{1}^{2} b_{0}, \\
R_{2}= & 2 \lambda a_{-1} b_{1}^{3}+2 c a_{1}^{3} b_{-1}-8 c^{3} a_{-1} b_{1}^{3} \\
& -2 c a_{1}^{2} a_{-1} b_{1}+8 c^{3} a_{1} b_{1}^{2} b_{-1} \\
& -2 \lambda a_{1} b_{1}^{2} b_{-1}-4 c^{3} a_{1} b_{1} b_{0}^{2} \\
& -2 \lambda a_{1} b_{1} b_{0}^{2}+2 c a_{1}^{2} a_{0} b_{0} \\
& -2 c a_{1} a_{0}^{2} b_{1}+4 c^{3} a_{0} b_{1}^{2} b_{0}+2 \lambda a_{0} b_{1}^{2} b_{0},
\end{aligned}
$$

$$
\begin{aligned}
R_{1}= & -\lambda a_{1} b_{0}^{3}-c a_{0}^{3} b_{1}+c^{3} a_{1} b_{0}^{3} \\
& -18 c^{3} a_{1} b_{1} b_{0} b_{-1}-6 \lambda a_{1} b_{1} b_{0} b_{-1} \\
& -6 c a_{1} a_{0} a_{-1} b_{1}+\lambda a_{0} b_{1}^{2} b_{-1} \\
& +c a_{1}^{2} a_{-1} b_{0}+\lambda a_{0} b_{1} b_{0}^{2} \\
& +c a_{0}^{2} a_{1} b_{0}-c^{3} a_{0} b_{1} b_{0}^{2} \\
& +23 c^{3} a_{0} b_{1}^{2} b_{-1}+5 c a_{1}^{2} a_{0} b_{-1} \\
& +5 \lambda a_{-1} b_{1}^{2} b_{0}-5 c^{3} a_{-1} b_{1}^{2} b_{0} \\
R_{0}= & 4 c a_{1}^{2} a_{-1} b_{-1}-4 c a_{1} a_{-1}^{2} b_{1} \\
& -4 c a_{0}^{2} a_{-1} b_{1}+4 c^{3} a_{1} b_{0}^{2} b_{-1} \\
& +32 c^{3} a_{-1} b_{1}^{2} b_{-1}-4 c^{3} a_{-1} b_{1} b_{0}^{2} \\
& -32 c^{3} a_{1} b_{1} b_{-1}^{2}-4 \lambda a_{1} b_{1} b_{-1}^{2} \\
& -4 \lambda a_{1} b_{0}^{2} b_{-1}+4 \lambda a_{-1} b_{1}^{2} b_{-1} \\
& +4 \lambda a_{-1} b_{1} b_{0}^{2}+4 c a_{1} a_{0}^{2} b_{-1}
\end{aligned}
$$$$
R_{-1}=c a_{0}^{3} b_{-1}-c^{3} a_{-1} b_{0}^{3}+\lambda a_{-1} b_{0}^{3}
$$$$
+6 \lambda a_{-1} b_{1} b_{0} b_{-1}+6 c a_{1} a_{-1} a_{0} b_{-1}
$$$$
+18 c^{3} a_{-1} b_{1} b_{0} b_{-1}-c a_{1} a_{-1}^{2} b_{0}
$$$$
+c^{3} a_{0} b_{-1} b_{0}^{2}-5 \lambda a_{1} b_{0} b_{-1}^{2}
$$$$
-c a_{0}^{2} a_{-1} b_{0}-5 c a_{0} a_{-1}^{2} b_{1}
$$$$
+5 c^{3} a_{1} b_{0} b_{-1}^{2}-\lambda a_{0} b_{-1} b_{0}^{2}
$$$$
-23 c^{3} a_{0} b_{1} b_{-1}^{2}-\lambda a_{0} b_{1} b_{-1}^{2} \text {, }
$$$$
R_{-2}=-2 c a_{0} a_{-1}^{2} b_{0}-4 c^{3} a_{0} b_{-1}^{2} b_{0}
$$$$
+2 \lambda a_{-1} b_{0}^{2} b_{-1}+2 c a_{0}^{2} a_{-1} b_{-1}
$$$$
+2 \lambda a_{-1} b_{1} b_{-1}^{2}+4 c^{3} a_{-1} b_{0}^{2} b_{-1}
$$$$
+2 c a_{-1}^{2} a_{1} b_{-1}-8 c^{3} a_{-1} b_{1} b_{-1}^{2}
$$$$
-2 \lambda a_{0} b_{-1}^{2} b_{0}+8 c^{3} a_{1} b_{-1}^{3}
$$$$
-2 \lambda a_{1} b_{-1}^{3}-2 c a_{-1}^{3} b_{1} \text {, }
$$$$
R_{-3}=\lambda a_{-1} b_{0} b_{-1}^{2}+c a_{-1}^{2} a_{0} b_{-1}
$$$$
-c^{3} a_{-1} b_{0} b_{-1}^{2}+c^{3} a_{0} b_{-1}^{3}
$$$$
-c a_{-1}^{3} b_{0}-\lambda a_{0} b_{-1}^{3} \text {. }
$$

Solving this system of algebraic equations by using symbolic computation, we obtain the following results. 
Case 1. We have

$$
\begin{gathered}
a_{0}=a_{0}, \quad a_{1}=-\frac{i \sqrt{6}\left(2 a_{0}^{2}+3 c^{2} b_{0}^{2}\right)}{24 c b_{-1}}, \\
a_{-1}=\frac{i \sqrt{6}}{2} c b_{-1}, \quad b_{0}=b_{0}, \\
b_{1}=\frac{2 a_{0}^{2}+3 c^{2} b_{0}^{2}}{12 c^{2} b_{-1}}, \quad b_{-1}=b_{-1}, \\
\lambda=-\frac{c^{3}}{2},
\end{gathered}
$$

where $a_{0}, b_{0}$, and $b_{-1}$ are free parameters. Substituting these results into (62), we obtain the following exact solution:

$$
\begin{aligned}
u(x, t)= & \left(-\frac{i \sqrt{6}\left(2 a_{0}^{2}+3 c^{2} b_{0}^{2}\right)}{24 c b_{-1}} \exp \left(c x-\frac{\lambda t^{\alpha}}{\Gamma(1+\alpha)}\right)+a_{0}\right. \\
& \left.+(i \sqrt{6} / 2) c b_{-1} \exp \left(-\left(c x-\frac{\lambda t^{\alpha}}{\Gamma(1+\alpha)}\right)\right)\right) \\
\times & \left(\frac{2 a_{0}^{2}+3 c^{2} b_{0}^{2}}{12 c^{2} b_{-1}} \exp \left(c x-\frac{\lambda t^{\alpha}}{\Gamma(1+\alpha)}\right)+b_{0}+b_{-1}\right. \\
& \left.\quad \times \exp \left(-\left(c x-\frac{\lambda t^{\alpha}}{\Gamma(1+\alpha)}\right)\right)\right)^{-1}
\end{aligned}
$$

Case 2. We have

$$
\begin{gathered}
a_{0}=a_{0}, \quad a_{1}=0, \quad a_{-1}=0, \\
b_{0}=0, \quad b_{1}=\frac{a_{0}^{2}}{24 c^{2} b_{-1}}, \\
b_{-1}=b_{-1}, \quad \lambda=c^{3},
\end{gathered}
$$

where $b_{-1}$ is a free parameter. Substituting these results into (62), we obtain the following exact solution:

$$
\begin{aligned}
u(x, t)=a_{0}( & \frac{a_{0}^{2}}{24 c^{2} b_{-1}} \exp \left(c x-\frac{c^{3} t^{\alpha}}{\Gamma(1+\alpha)}\right) \\
& \left.+b_{-1} \exp \left(-\left(c x-\frac{c^{3} t^{\alpha}}{\Gamma(1+\alpha)}\right)\right)\right)^{-1}
\end{aligned}
$$

If we set $a_{0}^{2}=24 c^{2}$ and $b_{-1}=1,(68)$ becomes

$$
u(x, t)=2 c \sqrt{6} \operatorname{sech}\left(c x-\frac{c^{3} t^{\alpha}}{\Gamma(1+\alpha)}\right)
$$

which is the exact solution of the time fractional fmKdV equation.
Case 3. We have

$$
\begin{gathered}
a_{0}=\frac{b_{0}\left(a_{-1}^{2}+3 c^{2} b_{-1}^{2}\right)}{a_{-1} b_{-1}}, \\
a_{1}=\frac{b_{0}^{2}\left(2 a_{-1}^{2}+3 c^{2} b_{-1}^{2}\right)}{8 a_{-1} b_{-1}^{2}}, \\
a_{-1}=a_{-1}, \\
b_{0}=b_{0}, \quad b_{1}=\frac{b_{0}^{2}\left(2 a_{-1}^{2}+3 c^{2} b_{-1}^{2}\right)}{8 a_{-1}^{2} b_{-1}}, \\
b_{-1}=b_{-1}, \quad \lambda=\frac{c\left(a_{-1}^{2}+c^{2} b_{-1}^{2}\right)}{b_{-1}^{2}},
\end{gathered}
$$

where $a_{-1}$ and $b_{-1}$ are free parameters. Substituting these results into (62), we obtain the following exact solution:

$$
\begin{aligned}
u(x, t)= & \frac{b_{0}^{2}\left(2 a_{-1}^{2}+3 c^{2} b_{-1}^{2}\right)}{8 a_{-1} b_{-1}^{2}} \exp \left(c x-\frac{\lambda t^{\alpha}}{\Gamma(1+\alpha)}\right) \\
& +\frac{b_{0}\left(a_{-1}^{2}+3 c^{2} b_{-1}^{2}\right)}{a_{-1} b_{-1}} \\
& \left.+a_{-1} \exp \left(-\left(c x-\frac{\lambda t^{\alpha}}{\Gamma(1+\alpha)}\right)\right)\right) \\
& \times\left(\frac{b_{0}^{2}\left(2 a_{-1}^{2}+3 c^{2} b_{-1}^{2}\right)}{8 a_{-1}^{2} b_{-1}} \exp \left(c x-\frac{\lambda t^{\alpha}}{\Gamma(1+\alpha)}\right)\right. \\
& \left.+b_{0}+b_{-1} \exp \left(-\left(c x-\frac{\lambda t^{\alpha}}{\Gamma(1+\alpha)}\right)\right)\right)^{-1}
\end{aligned}
$$

Case 4. We have

$$
\begin{gathered}
a_{0}=\frac{i \sqrt{6}}{2} c b_{0}, \quad a_{1}=-\frac{i \sqrt{6}}{2} c b_{1}, \\
a_{-1}=0, \quad b_{0}=b_{0}, \quad b_{1}=b_{1}, \\
b_{-1}=0, \quad \lambda=-\frac{c^{3}}{2},
\end{gathered}
$$

where $b_{0}$ and $b_{1}$ are free parameters. Substituting these results into (62), we obtain the following exact solution:

$$
\begin{aligned}
& u(x, t) \\
& \quad=\frac{i \sqrt{6} c}{2}\left(\frac{b_{1} \exp \left(c x+\left(c^{3} / 2\right) t^{\alpha} / \Gamma(1+\alpha)\right)-b_{0}}{b_{1} \exp \left(c x+\left(c^{3} / 2\right) t^{\alpha} / \Gamma(1+\alpha)\right)+b_{0}}\right) .
\end{aligned}
$$


If we set $b_{0}=1$ and $b_{1}=1,(73)$ becomes

$$
\begin{array}{r}
u(x, t)=\frac{i \sqrt{6} c}{2}\left(\sinh \left(c x+\frac{\left(c^{3} / 2\right) t^{\alpha}}{\Gamma(1+\alpha)}\right)\right. \\
\left.\quad+\cosh \left(c x+\frac{\left(c^{3} / 2\right) t^{\alpha}}{\Gamma(1+\alpha)}\right)-1\right) \\
\quad \times\left(\sinh \left(c x+\frac{\left(c^{3} / 2\right) t^{\alpha}}{\Gamma(1+\alpha)}\right)\right. \\
\left.\quad+\cosh \left(c x+\frac{\left(c^{3} / 2\right) t^{\alpha}}{\Gamma(1+\alpha)}\right)+1\right)^{-1}
\end{array}
$$

which is the exact solution of the time fractional fmKdV equation.

Case 5. We have

$$
\begin{gathered}
a_{0}=0, \quad a_{1}=-i \sqrt{6} c b_{1}, \quad a_{-1}=i \sqrt{6} c b_{-1}, \\
b_{0}=0, \quad b_{1}=b_{1}, \quad b_{-1}=b_{-1}, \\
\lambda=-2 c^{3},
\end{gathered}
$$

where $b_{1}$ and $b_{-1}$ are free parameters. Substituting these results into (62), we have the following exact solution:

$$
\begin{aligned}
u(x, t)=( & -i \sqrt{6} c b_{1} \exp \left(x+\frac{2 c^{3} t^{\alpha}}{\Gamma(1+\alpha)}\right) \\
& \left.+i \sqrt{6} c b_{-1} \exp \left(-\left(x+\frac{2 c^{3} t^{\alpha}}{\Gamma(1+\alpha)}\right)\right)\right) \\
& \times\left(b_{1} \exp \left(x+\frac{2 c^{3} t^{\alpha}}{\Gamma(1+\alpha)}\right)\right. \\
& \left.+b_{-1} \exp \left(-\left(x+\frac{2 c^{3} t^{\alpha}}{\Gamma(1+\alpha)}\right)\right)\right)^{-1} .
\end{aligned}
$$

If we take $b_{1}=b_{-1}=1,(76)$ becomes

$$
u(x, t)=-i c \sqrt{6} \tanh \left(x+\frac{2 c^{3} t^{\alpha}}{\Gamma(1+\alpha)}\right),
$$

which is the exact solution of the time fractional fmKdV equation.

The established solutions have been checked by putting them back into the original equation (52). To the best of our knowledge, they have not been obtained in literature.

\section{Conclusion}

In this paper, we use the Exp-function method to calculate the exact solutions for the time and space fractional nonlinear partial differential equations. When the parameters take certain values, the solitary wave solutions are derived from the exponential form. Since this method is very efficient, reliable, simple, and powerful in finding the exact solutions for the nonlinear fractional differential equations, the proposed method can be extended to solve many systems of nonlinear fractional partial differential equations. We hope that the present solutions may be useful in further numerical analysis and these results are going to be very useful in further future research.

\section{Conflict of Interests}

The authors declare that there is no conflict of interests regarding the publication of this paper.

\section{References}

[1] K. S. Miller and B. Ross, An Introduction to the Fractional Calculus and Fractional Differential Equations, Wiley, New York, NY, USA, 1993.

[2] I. Podlubny, Fractional Differential Equations, Academic Press, California, Calif, USA, 1999.

[3] A. A. Kilbas, H. M. Srivastava, and J. J. Trujillo, Theory and Applications of Fractional Differential Equations, Elsevier, Amsterdam, The Netherlands, 2006.

[4] A. M. A. El-Sayed and M. Gaber, "The Adomian decomposition method for solving partial differential equations of fractal order in finite domains," Physics Letters A, vol. 359, no. 3, pp. 175-182, 2006.

[5] M. Safari, D. D. Ganji, and M. Moslemi, "Application of He’s variational iteration method and Adomian's decomposition method to the fractional KdV-Burgers-Kuramoto equation," Computers and Mathematics with Applications, vol. 58, no. 11-12, pp. 2091-2097, 2009.

[6] N. H. Sweilam, M. M. Khader, and R. F. Al-Bar, "Numerical studies for a multi-order fractional differential equation," Physics Letters A, vol. 371, no. 1-2, pp. 26-33, 2007.

[7] M. Inc, "The approximate and exact solutions of the spaceand time-fractional Burgers equations with initial conditions by variational iteration method," Journal of Mathematical Analysis and Applications, vol. 345, no. 1, pp. 476-484, 2008.

[8] L. N. Song and H. Q. Zhang, "Solving the fractional BBMBurgers equation using the homotopy analysis method," Chaos, Solitons and Fractals, vol. 40, no. 4, pp. 1616-1622, 2009.

[9] A. A. M. Arafa, S. Z. Rida, and H. Mohamed, "Homotopy analysis method for solving biological population model," Communications in Theoretical Physics, vol. 56, no. 5, pp. 797800, 2011.

[10] K. A. Gepreel, “The homotopy perturbation method applied to the nonlinear fractional KolmogorovPetrovskiiPiskunov equations," Applied Mathematics Letters, vol. 24, no. 8, pp. 1428-1434, 2011.

[11] P. K. Gupta and M. Singh, "Homotopy perturbation method for fractional Fornberg-Whitham equation," Computers and Mathematics with Applications, vol. 61, no. 2, pp. 250-254, 2011.

[12] Z. Odibat and S. Momani, "A generalized differential transform method for linear partial differential equations of fractional order," Applied Mathematics Letters, vol. 21, no. 2, pp. 194-199, 2008.

[13] V. S. Ertürk, S. Momani, and Z. Odibat, "Application of generalized differential transform method to multi-order fractional 
differential equations," Communications in Nonlinear Science and Numerical Simulation, vol. 13, no. 8, pp. 1642-1654, 2008.

[14] A. Secer, M. Akinlar, and A. C. Cevikel, "Efficient solutions of systems of fractional PDEs by the differential transform method," Advances in Difference Equations, vol. 2012, p. 188, 2012.

[15] S. Zhang and H. Zhang, "Fractional sub-equation method and its applications to nonlinear fractional PDEs," Physics Letters A, vol. 375, no. 7, pp. 1069-1073, 2011.

[16] B. Tong, Y. He, L. Wei, and X. Zhang, "A generalized fractional sub-equation method for fractional differential equations with variable coefficients," Physics Letters A, vol. 376, no. 38-39, pp. 2588-2590, 2012.

[17] S. Guo, L. Mei, Y. Li, and Y. Sun, "The improved fractional sub-equation method and its applications to the space-time fractional differential equations in fluid mechanics," Physics Letters A, vol. 376, no. 4, pp. 407-411, 2012.

[18] B. Lu, "The first integral method for some time fractional differential equations," Journal of Mathematical Analysis and Applications, vol. 395, pp. 684-693, 2012.

[19] B. Zheng, "Exp-function method for solving fractional partial differential equations," The Scientific World Journal, vol. 2013, Article ID 465723, 8 pages, 2013.

[20] A. Bekir, Ö. Güner, and A. C. Cevikel, "Fractional complex transform and exp-function methods for fractional differential equations," Abstract and Applied Analysis, vol. 2013, Article ID 426462, 8 pages, 2013.

[21] B. Zheng, " $\left(G^{\prime} / G\right)$-expansion method for solving fractional partial differential equations in the theory of mathematical physics," Communications in Theoretical Physics, vol. 58, no. 5, pp. 623-630, 2012.

[22] K. A. Gepreel and S. Omran, "Exact solutions for nonlinear partial fractional differential equations," Chinese Physics B, vol. 21, no. 11, pp. 110-204, 2012.

[23] A. Bekir and Ö. Güner, "Exact solutions of nonlinear fractional differential equations by $\left(G^{\prime} / G\right)$-expansion method," Chinese Physics B, vol. 22, no. 11, pp. 110-202, 2013.

[24] J. H. He and X. H. Wu, "Exp-function method for nonlinear wave equations," Chaos, Solitons and Fractals, vol. 30, no. 3, pp. 700-708, 2006.

[25] J. H. He and M. A. Abdou, "New periodic solutions for nonlinear evolution equations using Exp-function method," Chaos, Solitons and Fractals, vol. 34, no. 5, pp. 1421-1429, 2007.

[26] A. Ebaid, "Exact solitary wave solutions for some nonlinear evolution equations via Exp-function method," Physics Letters A, vol. 365, no. 3, pp. 213-219, 2007.

[27] S. Kutluay and A. Esen, "Exp-function method for solving the general improved KdV equation," International Journal of Nonlinear Sciences and Numerical Simulation, vol. 10, no. 6, pp. 717-725, 2009.

[28] A. Bekir, "The exp-function method for Ostrovsky equation," International Journal of Nonlinear Sciences and Numerical Simulation, vol. 10, no. 6, pp. 735-739, 2009.

[29] S. Zhang, "Application of Exp-function method to highdimensional nonlinear evolution equation," Chaos, Solitons and Fractals, vol. 38, no. 1, pp. 270-276, 2008.

[30] A. Bekir and A. Boz, "Application of Exp-function method for $(2+1)$-dimensional nonlinear evolution equations," Chaos, Solitons and Fractals, vol. 40, no. 1, pp. 458-465, 2009.
[31] A. C. Cevikel and A. Bekir, "New solitons and periodic solutions for $(2+1)$ - dimensional Davey-Stewartson equations," Chinese Journal of Physics, vol. 51, pp. 1-13, 2013.

[32] S. Zhang, "Application of Exp-function method to a KdV equation with variable coefficients," Physics Letters A, vol. 365, no. 5-6, pp. 448-453, 2007.

[33] S. A. El-Wakil, M. A. Madkour, and M. A. Abdou, "Application of Exp-function method for nonlinear evolution equations with variable coefficients," Physics Letters A, vol. 369, no. 1-2, pp. 6269, 2007.

[34] S. D. Zhu, "Exp-function method for the Hybrid-Lattice system," International Journal of Nonlinear Sciences and Numerical Simulation, vol. 8, no. 3, pp. 461-464, 2007.

[35] A. Bekir, "Application of the Exp-function method for nonlinear differential-difference equations," Applied Mathematics and Computation, vol. 215, no. 11, pp. 4049-4053, 2010.

[36] C. Q. Dai and J. L. Chen, "New analytic solutions of stochastic coupled KdV equations," Chaos, Solitons and Fractals, vol. 42, no. 4, pp. 2200-2207, 2009.

[37] C. Q. Dai and J. F. Zhang, "Application of he's EXP-function method to the stochastic mKdV equation," International Journal of Nonlinear Sciences and Numerical Simulation, vol. 10, no. 5, pp. 675-680, 2009.

[38] G. Jumarie, "Modified Riemann-Liouville derivative and fractional Taylor series of nondifferentiable functions further results," Computers and Mathematics with Applications, vol. 51, no. 9-10, pp. 1367-1376, 2006.

[39] A. Ebaid, "An improvement on the Exp-function method when balancing the highest order linear and nonlinear terms," Journal of Mathematical Analysis and Applications, vol. 392, no. 1, pp. 15, 2012.

[40] L. N. Song, Q. Wang, and H. Q. Zhang, "Rational approximation solution of the fractional Sharma-Tasso-Olever equation," Journal of Computational and Applied Mathematics, vol. 224, no. 1, pp. 210-218, 2009.

[41] S. Momani, "Non-perturbative analytical solutions of the spaceand time-fractional Burgers equations," Chaos, Solitons and Fractals, vol. 28, no. 4, pp. 930-937, 2006.

[42] M. Kurulay and M. Bayram, "Approximate analytical solution for the fractional modified $\mathrm{KdV}$ by differential transform method," Communications in Nonlinear Science and Numerical Simulation, vol. 15, no. 7, pp. 1777-1782, 2010. 


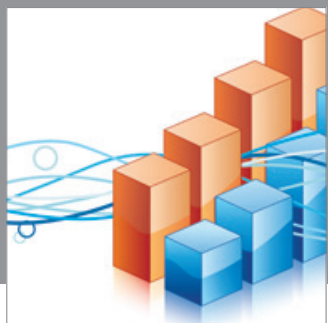

Advances in

Operations Research

mansans



The Scientific World Journal
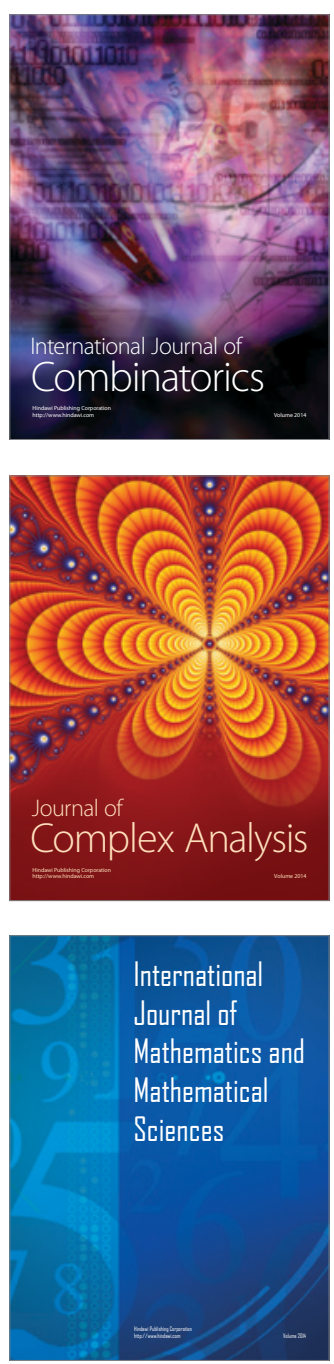
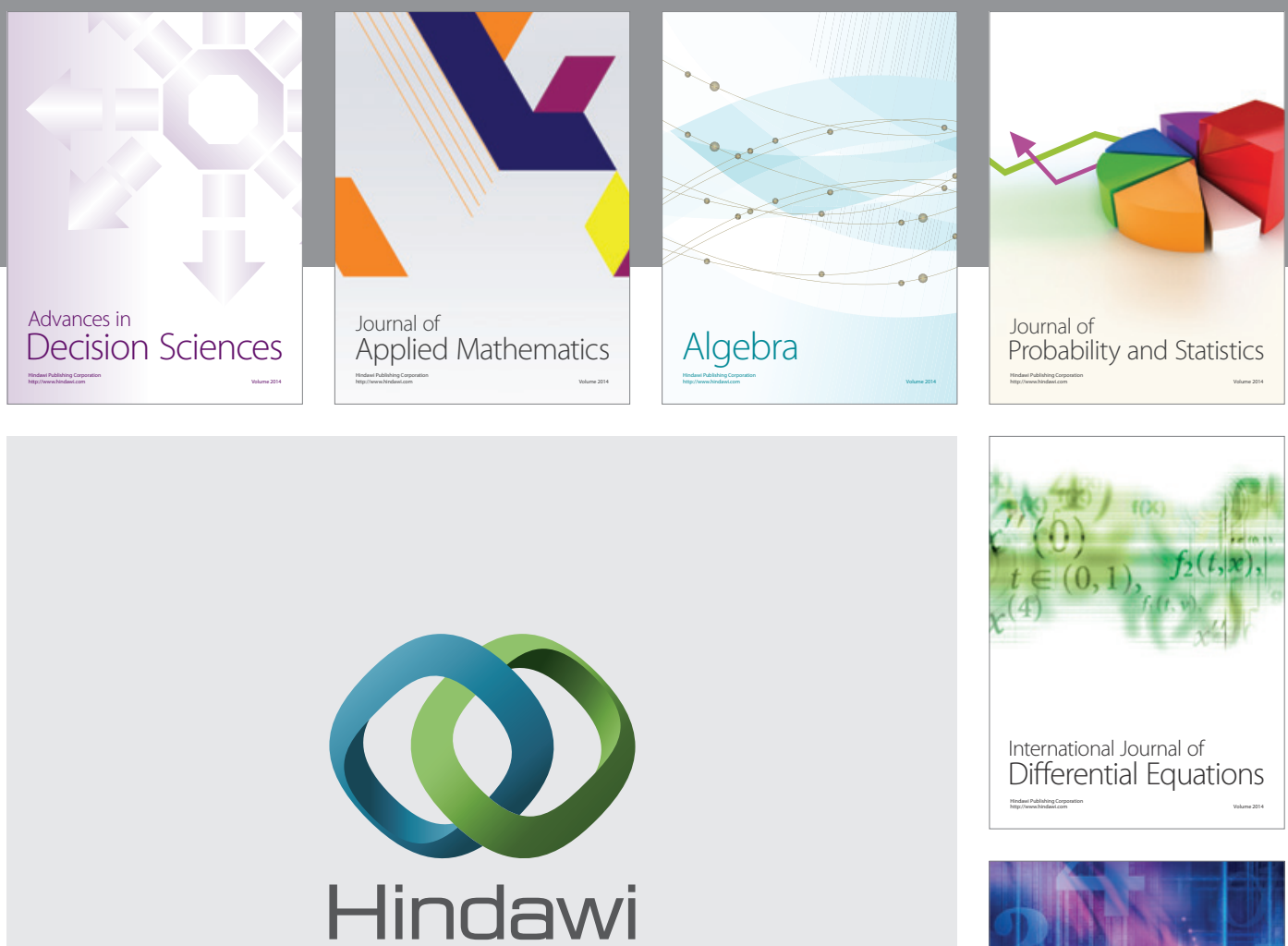

Submit your manuscripts at http://www.hindawi.com


Journal of

Function Spaces

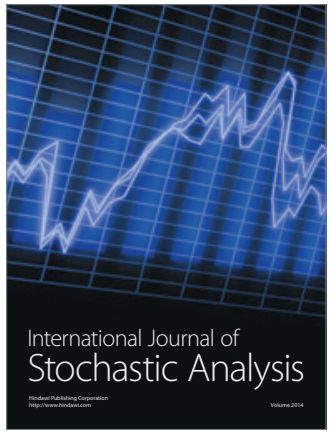


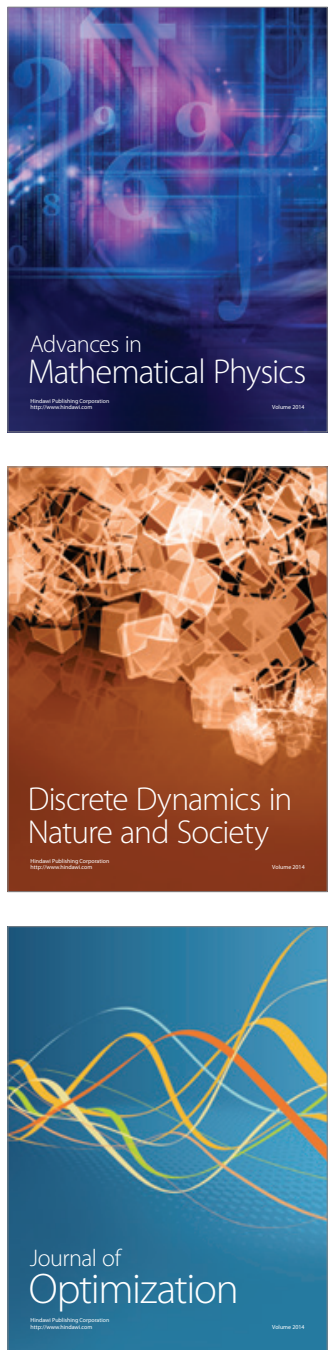